\title{
Knockdown of macrophage inhibitory cytokine-1 in RPMI-8226 human multiple myeloma cells inhibits osteoclastic differentiation through inhibiting the RANKL-Erk1/2 signaling pathway
}

\author{
MINGZHOU YUAN, JUNMIN CHEN and ZHIYONG ZENG \\ Department of Hematology and Rheumatology, The First Clinical Medical College of Fujian Medical University, \\ Fuzhou, Fujian 350005, P.R. China
}

Received April 8, 2015; Accepted February 25, 2016

DOI: $10.3892 / \mathrm{mmr} .2016 .5879$

\begin{abstract}
Patients with multiple myeloma (MM) often develop myeloma bone disease (MBD). The development of MBD from $\mathrm{MM}$ is considered to be caused by an abnormal bone marrow microenvironment. Macrophage inhibitory cytokine-1 (MIC-1) is a member of the transforming growth factor- $\beta$ superfamily. In patients with MM, MIC-1 is expressed at high levels, however, whether this increased expression of MIC-1 is associated with the development of MBD from MM remains to be elucidated. The present study investigated whether MIC-1 is essential for the osteoclastic differentiation of peripheral blood mononuclear cells (PBMNCs) by using a co-culture system, in which the PBMNCs were co-cultured with RPMI-8226 cells. The expression of MIC-1 in the RPMI-8226 cells was knocked down using RNA interference. Osteoclastic differentiation was evaluated using tartrate-resistant acid phosphatase staining and lacunar resorption on dentine slices. The expression of receptor activator of nuclear factor $-\kappa B$ ligand (RANKL) and phosphorylation of extracellular signal-regulated kinase (Erk)1/2 were measured using Western blotting. It was found that the reduced expression of MIC-1 in the RPMI-8226 cells inhibited the osteoclastic differentiation of PBMNCs and decreased the expression levels of RANKL and phosphorylated Erk1/2. It was concluded that MIC-1 promoted the osteoclastic differentiation of PBMNCs via the RANKL-Erk1/2 signaling pathway and, therefore, MIC-1 may offer potential as a target in the design of strategies to treat MBD.
\end{abstract}

Correspondence to: Professor Junmin Chen, Department of Hematology and Rheumatology, The First Clinical Medical College of Fujian Medical University, 20 Chazhong Road, Fuzhou, Fujian 350005, P.R. China

E-mail: drjunminchen@hotmail.com

Key words: macrophage inhibitory cytokine-1, multiple myeloma, myeloma bone disease, osteoclastogenesis

\section{Introduction}

Multiple myeloma (MM) is a malignant cancer of plasma cells, which represent $\sim 13 \%$ of all hematological malignancies (1), with $>80 \%$ of patients with MM developing myeloma bone disease (MBD). MBD is characterized by severe bone pain, vertebral compression fractures and pathological fractures, which are caused by osteoclastic bone resorption and impaired osteoblastic bone formation induced by the interaction between myeloma cells and the bone marrow microenvironment. However, the molecular mechanism by which MM leads to the development of MBD remains to be elucidated.

Genes that are expressed at high levels in the bone marrow of patients with MM have been identified (2), one of which is macrophage inhibitory cytokine-1 (MIC-1). MIC-1 belongs to the human transforming growth factor $\beta$ superfamily. Also known as growth differentiation factor-15, placental bone morphogenetic protein, prostate-derived factor and NSAID-activated gene-1 (3-6), MIC-1 has multiple functions. In a previous in vitro study, MIC-1 was shown to inhibit the activation of macrophages (7). MIC-1 may be an important factor in the development of several types of tumor, including MM (8). In patients with MM, MIC-1 is expressed at high levels in the mesenchymal stem cells of the bone marrow, and high levels of MIC-1 in the patients' serum predicts a poor prognosis (2). MIC-1 may be involved in osteoclastogenesis; in patients with prostate cancer with bone metastases, MIC-1 has been found to induce the maturation of osteoclasts (9). However, whether MIC-1 is involved in the development of MBD in patients with MM remains to be elucidated. In present study, in order to evaluate the effect of target inhibition of MIC-1 in RPMI-8226 cells on osteoclastic differentiation of peripheral blood mononuclear cells (PBMNCs) and the mechanisms by which MIC-1 contributes to the maturation of osteoclasts, a lentiviral RNAi system directed toward the MIC-1 gene was designed and constructed in RPMI-8226 cells. A co-culture system was used in the present study to determine the role of MIC-1 on osteoclastic differentiation. The results from the present study offered a potential strategy for the treatment of MBD in patients with MM. 


\section{Materials and methods}

Preparation of the lentiviral vector bearing MIC-1 short hairpin (sh)RNA. To understand the potential effect of MIC-1 in RPMI-8226 cells, an MIC-1 shRNA was designed to specifically knock down the gene expression of MIC-1. The MIC-1 shRNA was designed by Genechem (Shanghai, China), based on the MIC-1 cDNA sequence (GenBank accession no. AF019770.1; http://www.ncbi.nlm.nih.gov/nuccore/AF019770.1). A control shRNA encoding a nonspecific shRNA was used as a negative control. The MIC-1 shRNA and negative control shRNA were synthesized by Genechem (Shanghai, China). The synthesized oligonucleotides were designed, synthesized and inserted into a GV115 vector (GeneChem) to construct a lentiviral vector, according to the manufacturer's protocol, to generate lentiviral transfer plasmids: Lv-shRNA, bearing MIC-1 shRNA, and Lv-NC, bearing control shRNA as a negative control. The control shRNA had no significant homology to any human gene sequence. The lentiviral vectors were prepared by transfecting the Lv-shRNA or Lv-NC with packaging plasmids into 293T cells, as described previously (10).

Cell culture and lentiviral transduction. The RPMI-8226 cells (Wuhan University, Wuhan, China) were cultured in RPMI-1640 medium (Gibco; Thermo Fisher Scientific, Inc., Waltham, MA, USA) supplemented with $10 \%$ fetal calf serum (Gibco; Thermo Fisher Scientific, Inc.) at $37^{\circ} \mathrm{C}$ in a humidified atmosphere containing $5 \% \mathrm{CO}_{2}$. After 3 days, the RPMI-8226 cells were transduced with the lentiviral vectors at a multiplicity of infection of 15 in the presence of $2 \mu \mathrm{g} / \mathrm{ml}$ polybrene (Sigma-Aldrich, St. Louis, MO, USA). Following incubation at $37^{\circ} \mathrm{C}$ for $8 \mathrm{~h}$, the cells were washed twice with phosphate-buffered saline (PBS), and the mRNA and protein levels of MIC-1 in the cells were measured using reverse transcription-quantitative polymerase chain reaction (RT-qPCR) and Western blot analyses, respectively, to evaluate the viral infection efficiency.

$R T-q P C R$. Total RNA was extracted from the cells using TRIzol reagent (Invitrogen; Thermo Fisher Scientific, Inc.). The RNA $(4 \mu \mathrm{g})$ was reverse transcribed into cDNA using Thermoscript RT-PCR System reagent (Gibco; Thermo Fisher Scientific, Inc.), according to the manufacturer's protocol, prior for use in qPCR. The qPCR was performed on an Applied Biosystems PRISM 7300 sequence detection system (Applied Biosystems; Thermo Fisher Scientific, Inc.) using a SYBR Green PCR master mix (DRR041A; Takara Bio, Inc., Tokyo, Japan). A total volume of $20 \mu \mathrm{l}$, containing $2 \mu \mathrm{l}$ cDNA, $10 \mu \mathrm{l}$ SYBR Premix Ex Taq, $0.4 \mu 1$ Forward Primer, $0.4 \mu 1$ Reverse Primer, $0.4 \mu \mathrm{l}$ ROX Reference Dye II and $6.8 \mu \mathrm{l} \mathrm{dH} 2 \mathrm{O}$, was used in the q-PCR reaction. Each sample was analyzed in triplicate. The quantification cycle $(\mathrm{Cq})$ value of each sample was normalized to an endogenous control, GAPDH. The $2^{-\Delta \Delta C q}$ method was used to relatively quantify the mRNA levels of MIC-1 (11). The primer (Genechem) sequences for human MIC-1 were: Sense 5'-GTTGCGGAAACGCTACGA-3' and antisense 5'-AACAGAGCCCGGTGAAGG-3'. Primers for the control (GAPDH) were: Sense 5'-TGACTTCAACAGCGA CACCCA-3' and antisense 5'-CACCCTGTTGCTGTAGCC AAA-3'. The qPCR program was as follows: 45 cycles of $95^{\circ} \mathrm{C}$

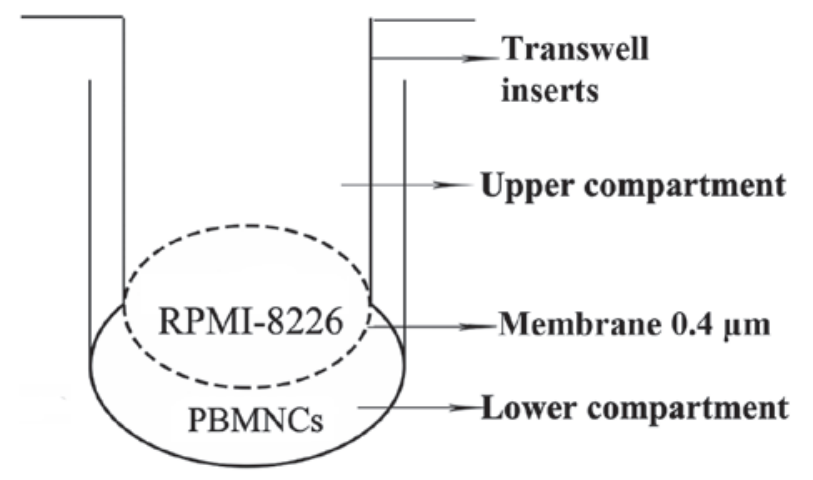

Figure 1. Schematic diagram of the Transwell system used to co-culture the PBMNCs and RPMI-8226 cells. PBMNCs, peripheral blood mononuclear cells.

for $15 \mathrm{sec}, 60^{\circ} \mathrm{C}$ for $30 \mathrm{sec}$ and $72^{\circ} \mathrm{C}$ for $45 \mathrm{sec}$, followed by $72^{\circ} \mathrm{C}$ for $10 \mathrm{~min}$.

Isolation and culture of PBMNCs. The PBMNCs were isolated, as described previously (10) from the peripheral blood of healthy donors, who had provided written informed consent. The present study was approved by the Ethics Committee of Fujian Medical University. A total of $10 \mathrm{ml}$ human blood was obtaubed from peripheral blood samples, and was mixed with $10 \mathrm{ml}$ lymphocyte separation medium (Hao Yang Biological Manufacture Co. Ltd., Tianjin, China) in a centrifuge tube. The mixture was centrifuged at $2,000 \mathrm{xg}$ at room temperature for 20 mins. Following washing with PBS once and $\alpha$-minimal essential medium ( $\alpha$-MEM) twice, the nucleated cells were grown in $\alpha$-MEM supplemented with $10 \%$ (v/v) fetal bovine serum (FBS), $50 \mathrm{ng} / \mathrm{ml}$ macrophage-colony-stimulating factor (M-CSF; PeproTech, Rocky Hill, NJ, USA) and $100 \mathrm{ng} / \mathrm{ml}$ receptor activator of nuclear factor- $\mathrm{KB}$ ligand (RANKL; Peprotech) at $37^{\circ} \mathrm{C}$ in $5 \% \mathrm{CO}_{2}$ humidified air. The medium was replaced every 3 days.

Co-culture system. To investigate the potential effect of MIC-1 on the differentiation capacity of the PBMNCs, a Transwell insert system (Corning, Inc., New York, NY, USA) was used (Fig. 1) to co-culture the PBMNCs $\left(1 \times 10^{6}\right.$ cells/well) and the RPMI- 8226 cells $\left(2 \times 10^{3}\right.$ cells/well) transduced with Lv-shRNA or Lv-NC. The PBMNCs were seeded into the lower compartment following placement of a cover glass and dentine slides on the bottom. The cells were cultured in $\alpha$-MEM (Gibco; Thermo Fisher Scientific, Inc.) containing 10\% FBS, $50 \mathrm{ng} / \mathrm{ml}$ M-CSF and $100 \mathrm{ng} / \mathrm{ml} \mathrm{RANKL}$. After $12 \mathrm{~h}$ of culture at $37^{\circ} \mathrm{C}$ in $5 \% \mathrm{CO}_{2}$, the non-adherent cells in the culture were removed by gently washing with fresh medium; the RPMI-8226 cells transduced with Lv-shRNA or Lv-NC were cultured in the upper compartment. RPMI-8226 cells without viral transduction were also used as a control.

Tartrate-resistant acid phosphatase (TRAP) staining and resorption pitformationassay. The PBMNCs $\left(1 \times 10^{6}\right.$ cells/well $)$, which were cultured on cover glasses and dentine slides for 14 or 21 days, were stained using a leukocyte acid phosphatase kit (Sigma-Aldrich), and the cells were then counterstained with hematoxylin (Sigma-Aldrich), as previously described (10). A 
mature osteoclast was identified as a cell with at least three TRAP-positive nuclei. To determine the number of mature osteoclasts in each group, 10 randomly selected fields from the three cultures in each group were examined under a microscope (BX43; Olympus, Tokyo, Japan). For the resorption pit assay, the cells on dentine slices were stained with toluidine blue and then scanned under a microscope (BX43; Olympus). Osteoclastic bone resorption was measured, based on the area of resorption pits per field from three cultures in each group.

Western blot analysis. The PBMNCs were harvested after co-culture for 14 days for protein analysis. The protein extracts from these cells were separated by $6-15 \%$ SDS-PAGE gel electrophoresis and then transferred onto polyvinylidene fluoride (PVDF; Beyotime Institute of Biotechnology, Shanghai, China) membranes. Following blocking with Tris-buffered saline with $0.2 \%$ Tween (TBST) containing 5\% non-fat milk at $4^{\circ} \mathrm{C}$ overnight, the membranes were incubated with the following antibodies diluted in TBST for $2 \mathrm{~h}$ at $4^{\circ} \mathrm{C}$ : Rabbit anti-RANKL (cat no. sc-9073; 1:2,000), rabbit anti-GAPDH (cat no. sc-25778; 1:2,000), rabbit anti-c-fos (cat no. sc-52, $1: 2,000)$, rabbit anti-c-Jun N-terminal kinase (c-JNK; cat no. sc-572; 1:2,000), rabbit anti-p-c-Jun N-terminal kinase (p-c-JNK; cat no. sc-135642; 1:2,000), rabbit anti-P-38 (cat no. sc-535; 1:2,000), rabbit anti-p-P-38 (cat no. sc-101759; $1: 2,000)$, rabbit anti-c-jun (cat no. sc-44; 1:2,000), rabbit anti-p-c-jun (cat no. sc-7980-R; 1:2,000), all purchased from Santa Cruz Biotechnology, Inc. (Santa Cruz, CA, USA), and rabbit anti-extracellular signal-regulated kinase 1/2 (Erk1/2; cat no. 4695; 1:2,000; Cell Signaling Technology, Inc., Danvers, MA, USA) or rabbit anti- p-Erk1/2 (cat no. 4376; 1:2,000; Cell Signaling Technology, Danvers, MA, USA). Following washing three times with TBST ( $\mathrm{pH} 7.5)$, the membranes were incubated with horseradish peroxidase-conjugated goat anti-rabbit secondary antibody (cat no. bs-0295G; 1:1,000; Beijing Biosynthesis Biotechnology, Beijing, China) for $1 \mathrm{~h}$ at room temperature. The membranes were washed three times with TBST ( $\mathrm{pH}$ 7.5). The resulting protein bands were visualized using an enhanced chemiluminescence reagent kit (Beyotime Institute of Biotechnology).

Statistical analysis. Statistical analysis was performed using SPSS 16.0 software (SPSS, Inc., Chicago, IL, USA). The data are expressed as the mean \pm standard deviation. One-way analysis of variance and Student-Newman-Keuls analysis were used to evaluate the statistical significance of differences among the groups. $\mathrm{P}<0.05$ was considered to indicate a statistically significant difference.

\section{Results}

Expression of MIC-1 in RPMI-8226 cells. To determine whether MIC-1 shRNA successfully knocked down the gene expression of MIC-1, the present study prepared lentiviral vectors bearing either MIC-1 shRNA (Lv-shRNA) or non-specific sequence ( $\mathrm{Lv}-\mathrm{NC}$ ). Following transduction with Lv-shRNA or Lv-NC for 14 days, the RPMI-8226 cells were harvested and the expression levels of MIC-1 in these cells were analyzed using RT-qPCR and Western blot analyses. As shown in Fig. 2, the mRNA and protein levels of MIC-1 were significantly lower in the cells transduced with Lv-shRNA, compared with those in the cells transduced with Lv-NC or those without viral tranduction.

MIC-1 is essential for the osteoclastic differentiation of $P B M N C$ s. To investigate the role of MIC-1 in the osteoclastic differentiation of PBMNCs, the PBMNCs were stained for TRAP following co-culture with RPMI-8226 cells for 14 days. The number of TRAP(+) cells was significantly lower when the PBMNCs were co-cultured with the RPMI-8226 cells transduced with Lv-shRNA, compared with when they were co-cultured with RPMI-8226 cells transduced with Lv-NC or without viral transduction (Fig. 3A and B). To investigate the role of MIC-1 in bone resorption by differentiated PBMNCs, the PBMNCs were stained with toluidine blue following co-culture with RPMI-8226 cells for 14 days. The percentage of pit area within a randomly selected area was significantly lower when the PBMNCs were co-cultured with the RPMI-8226 cells transduced with Lv-shRNA, compared with those co-cultured with the RPMI-8226 cells transduced with Lv-NC or without viral transduction (Fig. 3C and D). Taken together, these results suggested that MIC-1 was required for the osteoclastic differentiation and bone resorption activities of PBMNCs.

To confirm the role of MIC-1 in the osteoclastic differentiation of PBMNCs, the present study examined whether the expression of the osteoclast-specific gene, RANKL, was altered by the decreased expression of MIC-1. As shown in Fig. 4A and B, the protein level of RANKL in the PBMNCs was significantly lower when the cells were co-cultured with the RPMI-8226 cells transduced with Lv-shRNA, compared with those co-cultured with the RPMI-8226 cells transduced with Lv-NC or without viral transduction.

Subsequently, the present study investigated which signaling pathway was activated by RANKL by analyzing the phosphorylation status of the ERK1/2, c-JNK and p38 mitogen-activated protein kinase (MAPK) proteins using Western blot analysis. It was found that the protein levels of phosphorylated Erk1/2, but not those of c-JNK or p38, were significantly lower in the PBMNCs co-cultured with the RPMI-8226 cells transduced with Lv-shRNA, compared with those co-cultured with RPMI-8226 cells transduced with Lv-NC or without viral transduction (Fig. 4C and D), indicating that the MCI-1-induced increase in RANKL may activate the Erk1/2 pathway.

The present study then investigated whether c-fos and c-jun, important downstream molecules of ERK1/2, were involved in the differentiation of osteoclasts by analyzing the phosphorylation status of c-fos and c-jun using Western blot analysis. It was found that the protein levels of total c-fos were markedly reduced in the PBMNCs co-cultured with the RPMI-8226 cells transduced with Lv-shRNA, compared with those co-cultured with the RPMI-8226 cells transduced with $\mathrm{Lv}-\mathrm{NC}$ or without viral transduction. However, no changes were observed in the protein levels of total c-jun or phosphorylated c-jun in the PBMNCs co-cultured with the RPMI-8226 cells transduced with either Lv-shRNA or Lv-NC (Fig. 4C and D). Taken together, these results suggested that MIC-1 regulated the osteoclastic differentiation and bone resorption activities of the PBMNCs via the Erk1/2 signaling pathway. 


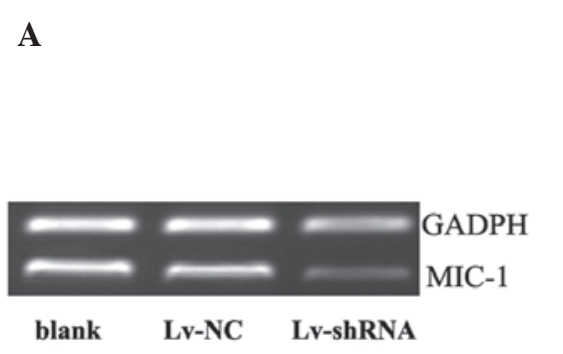

B

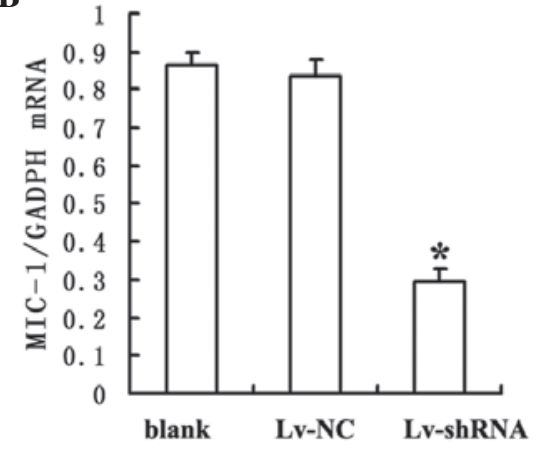

D

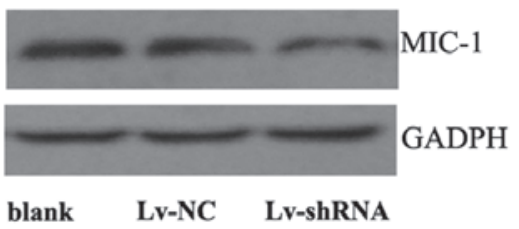

$\mathbf{E}$
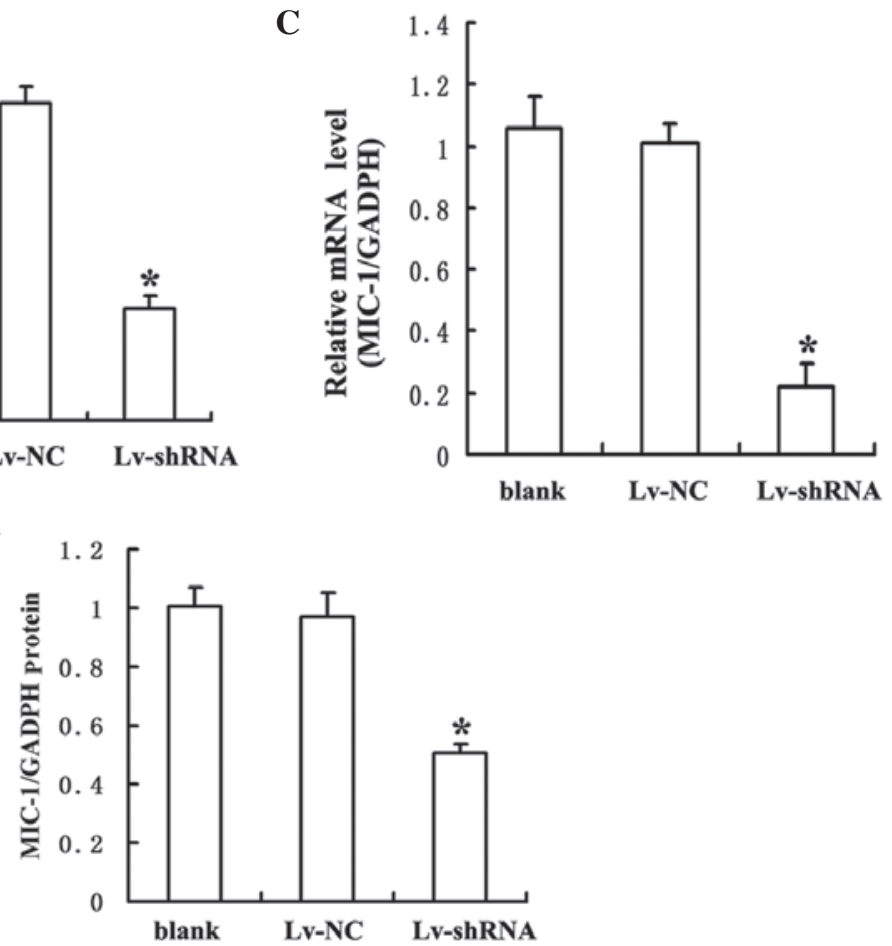

Figure 2. MIC-1 shRNA decreases the expression of MIC-1 in RPMI-8226 cells. RPMI-8226 cells were transduced with Lv-shRNA or Lv-NC. At day 14-post transduction, the cells were harvested and the expression of MIC-1 was analyzed. (A and B) The relative mRNA levels were analyzed by RT-qPCR, (C) real-tim PCR analysis of the mRNA expression levels of MIC-1 and (D and E) western blot analysis to assess protein expression levels. were performed GADPH was used as an internal control for each analysis. Data are presented as the mean \pm standard deviation. ${ }^{*} \mathrm{P}<0.05$, vs. Lv-NC or blank group. Blank, cells without viral transduction; Lv-NC, cells transduced with Lv-NC; Lv-shRNA, cells transduced with Lv-shRNA. shRNA, short hairpin RNA; NC, negative control; MIC-1, macrophage inhibitory cytokine-1.

A
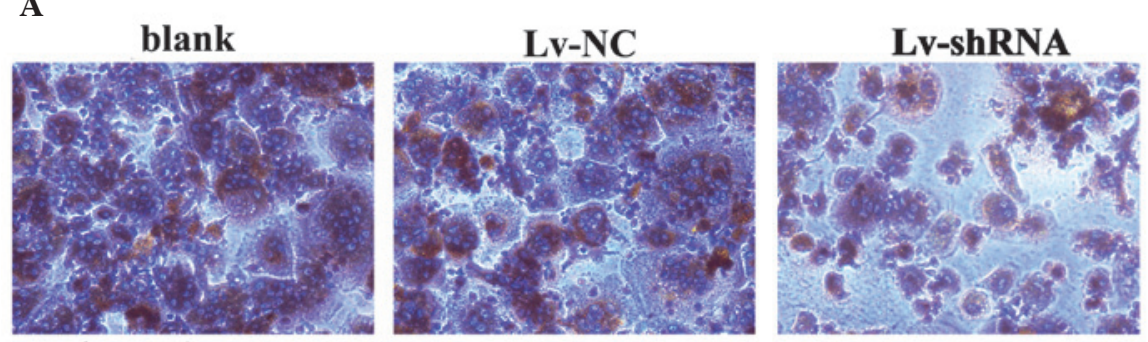

C

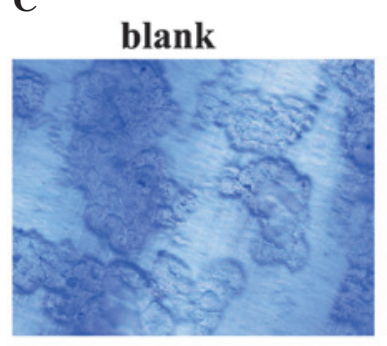

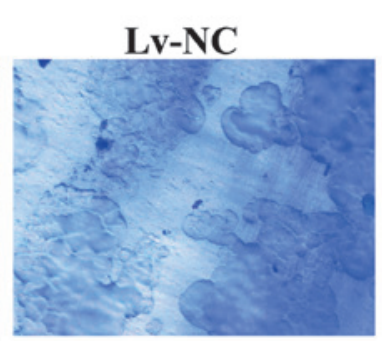

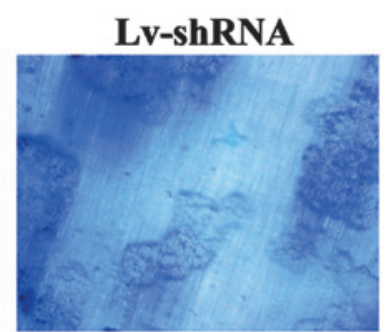

B

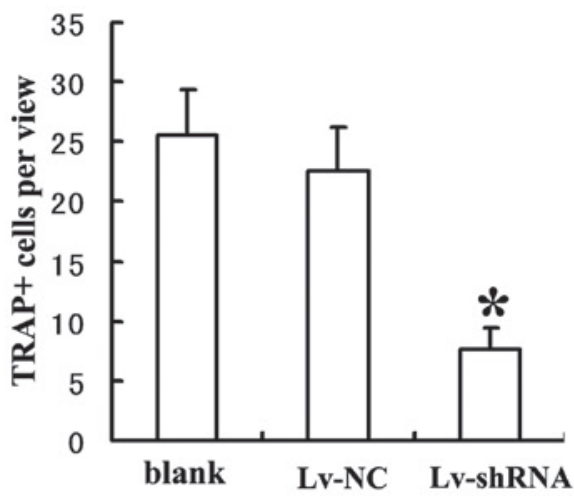

D

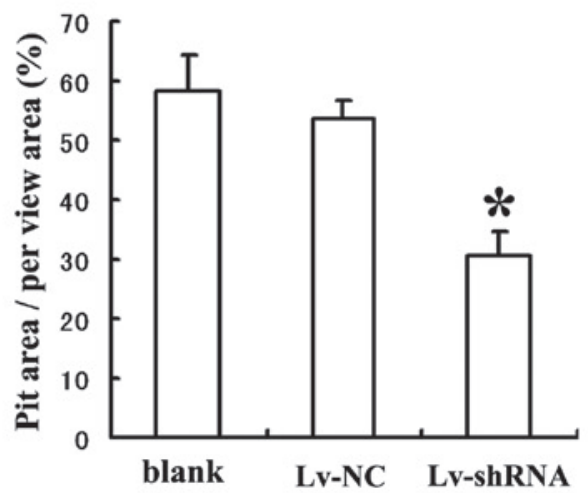

Figure 3. Osteoclastic differentiation of PBMNCs co-cultured with RPMI-8226 cells. PBMNCs were co-cultured with RPMI-8266 cells infected with Lv-shRNA or Lv-NC. At day 14-post infection, the PBMNCs were stained with (A) TRAP (magnification, x400) and the (B) numbers of TRAP-positive cells were counted per randomly selected field. (C) Toluidine blue staining (magnification, x200) was performed for the resorption pit assay, and the (D) resorption pit area per randomly selected field was calculated. Data are presented as the mean \pm standard deviation. " $\mathrm{P}<0.05$, vs. Lv-NC or blank group. shRNA, short hairpin RNA; NC, negative control; MIC-1, macrophage inhibitory cytokine-1; TRAP, tartrate-resistant acid phosphatase. 
A

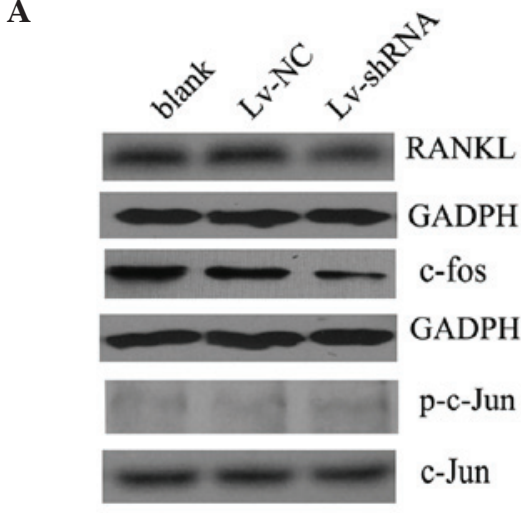

C

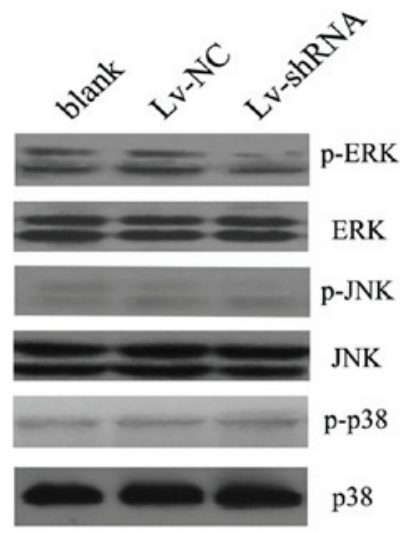

B

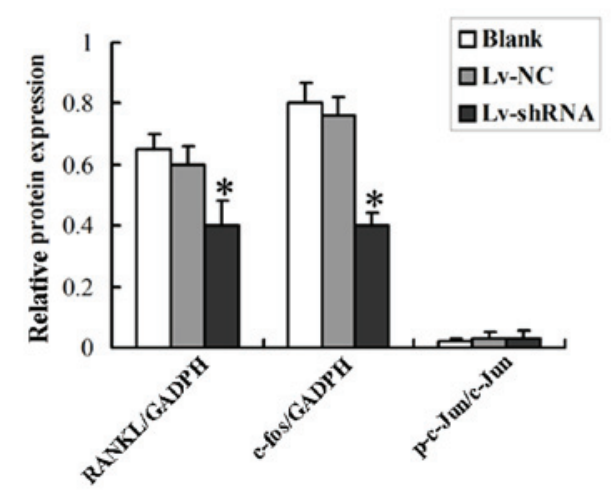

D

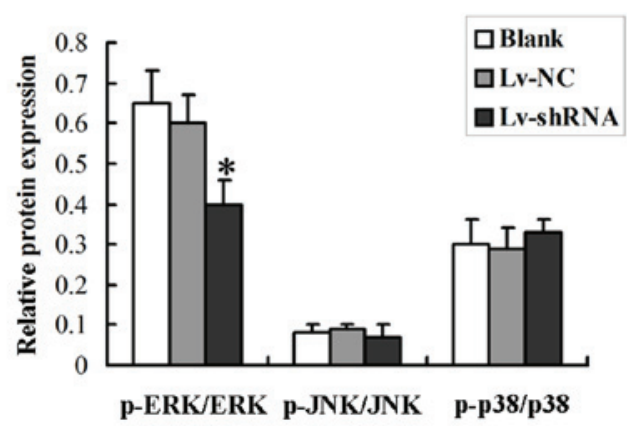

Figure 4. Expression of RANKL and the phosphorylation status of the Erk1/2 signaling pathway in PBMNCs. PBMNCs were co-cultured with RPMI-8226 cells infected with Lv-shRNA or Lv-NC for 14 days prior to harvesting for the analysis of the expression levels of (A) RANKL and c-fos, and the phosphorylation status of c-jun. GADPH was used as internal control. (B) Densitometric analysis of the western blot was performed. (C) The phosphorylation status of Erk1/2, p-38 and c-JNK was determined by western blotting. (D) Densitometric analysis of the western blot was performed. The data are expressed as the mean \pm standard deviation ("P<0.05 vs. Lv-NC group or Blank group). Blank, cells without viral transduction; PBMNCs, peripheral blood mononuclear cells; shRNA, short hairpin RNA; NC, negative control; RANKL, receptor activator of nuclear factor-kB ligand; p-ERK, phosphorylated-extracellular signal-regulated kinase; t-ERK, total ERK; JNK, c-Jun N-terminal kinase.

\section{Discussion}

In the present study, the role of MIC-1 in the osteoclastic differentiation of PBMNCs was examined. A co-culture system was used, in which PBMNCs co-cultured with RPMI-8226 cells were induced to differentiate into osteoclasts and to resorb bone. To knock down the expression of MIC-1 in the RPMI-8226 cells, a lentiviral vector was used to deliver the MIC-1 shRNA to the RPMI-8226 cells. It was found that MIC-1 shRNA efficiently decreased the expression of MIC-1 in the RPMI-8226 cells (Fig. 2). The present study then demonstrated that reduced expression of MIC-1 inhibited the osteoclastic differentiation of PBMNCs (Fig. 3). Finally, it was shown that the expression of the osteoclast-specific gene, RANKL, and the levels of dephosphorylated Erk1/2 were decreased in those PBMNCs, which were co-cultured with the RPMI-8226 cells transduced with Lv-shRNA (Fig. 4A and C). These results led to the conclusion that MIC-1 promoted osteoclastic differentiation of PBMNCs through inhibition of the RANKL-Erk1/2 signaling pathway.

Patients with MM often develop MBD with osteoclastic differentiation, induced by cytokines and other soluble factors in the bone marrow (12). In the bone marrow of patients with MM, MIC-1 is expressed at high levels (2). It has been suggested that MIC-1 may be involved in the regulation of osteoclastogenesis $(13,14)$. The results of the study demonstrated that MIC-1 was required in order for RPMI-8226 to induce osteoclastic differentiation of the PBMNCs (Figs. 3 and 4), supporting the role of MCI-1 in the development of MBD in patients with MM.

The osteoclast-specific marker gene, RANKL, is involved in MCI-1 stimulated osteoclastic differentiation. Binding to its receptor RANK, RANKL stimulates the osteoclastic differentiation of monocyte macrophages and the maturation of osteoclasts (15). In RANKL-deficient mice, severe osteopetrosis and a defect in tooth eruption have been observed (16). In patients with MM, RANKL has been shown to be involved osteoclastic differentiation (17-19). In the present study, it was shown that the expression of RANKL in the PBMNCs was reduced when the expression of MIC-1 in the co-cultured RPMI-8226 cells was knocked down (Fig. 4). RANKL binds to RANK and activates several MAPK signaling pathways (20), including the Erk1/2, JNK and P-38 pathways (21). The present study demonstrated that reduced expression of MIC-1 in the RPMI-8226 cells appeared to decrease the phosphorylation of Erk1/2, but not JNK or P-38, indicating the involvement of the Erk1/2 pathway in MIC-1-induced osteoclastic differentiation of PBMNCs $(22,23)$. Consistent with this observation, the 
expression levels of c-fos and c-jun, downstream molecules of the Erk1/2 signaling pathway in PBMNCs, were also decreased when the expression of MCI-1 in the co-cultured RPMI-8226 cells was reduced.

In conclusion, the present study found that MIC-1 was involved in the development of MBD in patients with MM by promoting the osteoclastic differentiation of PBMNCs by activating the RANKL-Erk1/2 signaling pathway. Thus, MIC-1 may offer potential as a target gene in the development of strategies to treat MM.

\section{References}

1. Kyle RA and Rajkumar SV: Criteria for diagnosis, staging, risk stratification and response assessment of multiple myeloma. Leukemia 23: 3-9, 2009.

2. Corre J, Mahtouk K, Attal M, Gadelorge M, Huynh A, Fleury-Cappellesso S, Danho C, Laharrague P, Klein B, Rème T and Bourin P: Bone marrow mesenchymal stem cells are abnormal in multiple myeloma. Leukemia 21: 1079-1088, 2007.

3. Hromas R, Hufford M, Sutton J, Xu D, Li Y and Lu L: PLAB, a novel placental bone morphogenetic protein. Biochim Biophys Acta 1354: 40-44, 1997.

4. Paralkar VM, Vail AL, Grasser WA, Brown TA, Xu H, Vukicevic S, Ke HZ, Qi H, Owen TA and Thompson DD: Cloning and characterization of a novel member of the transforming growth factor-beta/bone morphogenetic protein family. J Biol Chem 273: 13760-13767, 1998.

5. Böttner M, Suter-Crazzolara C, Schober A and Unsicker K: Expression of a novel member of the TGF-beta superfamily, growth/differentiation factor-15/macrophage-inhibiting cytokine-1 (GDF-15/MIC-1) in adult rat tissues. Cell Tissue Res 297: 103-110, 1999.

6. Baek SJ, Horowitz JM and Eling TE: Molecular cloning and characterization of human nonsteroidal anti-inflammatory drug-activated gene promoter. Basal transcription is mediated by Spl and Sp3. J Biol Chem 276: 33384-33392, 2001.

7. Bootcov MR, Bauskin AR, Valenzuela SM, Moore AG, Bansal M, He XY, Zhang HP, Donnellan M, Mahler S, Pryor K, et al: Mic-1, a novel macrophage inhibitory cytokine, is a divergent member of the TGF-beta superfamily. Proc Natl Acad Sci USA 94 11514-11519, 1997.

8. Tarkun P, Birtas Atesoglu E, Mehtap O, Musul MM and Hacihanefioglu A: Serum growth differentiation factor 15 levels in newly diagnosed multiple myeloma patients. Acta Haematol 131: 173-178, 2014.
9. Wakchoure S, Swain TM, Hentunen TA, Bauskin AR, Brown DA, Breit SN, Vuopala KS, Harris KW and Selander KS: Expression of macrophage inhibitory cytokine-1 in prostate cancer bone metastases induces osteoclast activation and weight loss. Prostate 69: 652-661, 2009.

10. Zeng Z, Zhang C and Chen J: Lentivirus-mediated RNA interference of DC-STAMP expression inhibits the fusion and resorptive activity of human osteoclasts. J Bone Miner Metab 31: 409-416, 2013.

11. Livak KJ and Schmittgen TD: Analysis of relative gene expression data using real-time quantitative PCR and the 2(-Delta Delta C(T)) Method. Methods 25: 402-408, 2001.

12. Roodman GD: Pathogenesis of myeloma bone disease. Leukemia 23: 435-441, 2009.

13. Khosla S: Minireview: The OPG/RANKL/RANK system. Endocrinology 142: 5050-5055, 2001.

14. Sung B, Prasad S, Yadav VR, Gupta SC, Reuter S, Yamamoto N, Murakami A and Aggarwal BB: RANKL signaling and osteoclastogenesis is negatively regulated by cardamonin. PLoS One 8: e64118, 2013.

15. Lacey DL, Timms E, Tan HL, Kelley MJ, Dunstan CR, Burgess T, Elliott R, Colombero A, Elliott G, Scully S, et al: Osteoprotegerin ligand is a cytokine that regulates osteoclast differentiation and activation. Cell 93: 165-176, 1998.

16. Kong YY, Yoshida H, Sarosi I, Tan HL, Timms E, Capparelli C, Morony S, Oliveira-dos-Santos AJ, Van G, Itie A, et al: OPGL is a key regulator of osteoclastogenesis, lymphocyte development and lymph-node organogenesis. Nature 397: 315-323, 1999.

17. Schramek D, Leibbrandt A, Sigl V, Kenner L, Pospisilik JA, Lee HJ, Hanada R, Joshi PA, Aliprantis A, Glimcher L, et al: Osteoclast differentiation factor RANKL controls development of progestin-driven mammary cancer. Nature 468: 98-102, 2010.

18. Sung B, Oyajobi B and Aggarwal BB: Plumbagin inhibits osteoclastogenesis and reduces human breast cancer-induced osteolytic bone metastasis in mice through suppression of RANKL signaling. Mol Cancer Ther 11: 350-359, 2012.

19. Ai LS, Sun CY, Zhang L, Zhou SC, Chu ZB, Qin Y, Wang YD, Zeng W, Yan H, Guo T, et al: Inhibition of BDNF in multiple myeloma blocks osteoclastogenesis via down-regulated stroma-derived RANKL expression both in vitro and in vivo. PLoS One 7: e46287, 2012.

20. Wada T, Nakashima T, Hiroshi N and Penninger JM: RANKL-RANK signaling in osteoclastogenesis and bone disease. Trends Mol Med 12: 17-25, 2006.

21. Huang P, Han J and Hui L: MAPK signaling in inflammation-associated cancer development. Protein Cell 1: 218-226, 2010.

22. Zhang W and Liu HT: MAPK signal pathways in the regulation of cell proliferation in mammalian cells. Cell Res 12: 9-18, 2002.

23. Feng X: RANKing intracellular signaling in osteoclasts. IUBMB Life 57: 389-395, 2005. 